\title{
ON RHODES EXPANSIONS OF BANDS
}

\author{
by H. SEZINANDO*
}

(Received 18th May 1994)

\begin{abstract}
We present a direct computation of the Rhodes expansions of the free objects in the varieties of bands, based in the manipulation of the invariants $i_{n}$ introduced by Gerhard and Petrich [4] in the study of bands.
\end{abstract}

1991 Mathematics subject classification: primary 20M10, secondary $20 \mathrm{M} 17$.

\section{Introduction}

In [8] Reilly studied the Rhodes expansions of completely regular semigroups, using techniques deriving from the work of Polák $[5,6,7]$. Here we give an alternative and quite different computation of the Rhodes expansion of $S$ in the special case where $S$ is a free object in a variety of bands. This is based on manipulation of the invariants $i_{n}$, introduced by Gerhard and Petrich [4] and on results obtained by the author [10] concerning the cardinalities of the free objects in varieties of bands. The work is part of the author's Ph.D. thesis [9].

\section{Preliminaries}

We record here the notation to be used throughout the paper and we state some theorems to be used later.

$X$ : a fixed countably infinite set. Elements of $X$ are called variables.

$A$ : a set, called an alphabet. Elements of $A$ are called letters.

$A^{*}$ : the free monoid on $A$. Elements of $A^{*}$ are called words. They are finite strings of elements of $A$ written as $a_{1} \cdots a_{n}$, where $a_{1}, \ldots a_{n} \in A$. The product is concatenation. The identity of $A^{*}$ is denoted by 1 . It is thought of as the empty string.

$c(w)$ : the content of $w \in A^{*}$ is the set of letters occurring $w$. By definition, $c(1)=\phi$.

$\bar{w}$ : the dual of $w$ is the word obtained from $w$ by reversing the order of the variables. That is, if $w=a_{1} \cdots a_{n}$ with $a_{1} \cdots a_{n} \in A$, then $\bar{w}=a_{n} \cdots a_{1}$.

$\bar{V}$ : the dual variety of $V$.

$F_{A}(V)$ : the free object of the variety $V$, on the set of generators $A$.

$[u=v]$ : the variety of semigroups defined by the equations $u=v, x^{2}=x$.

\footnotetext{
* This work was partially supported by S.A.L. (JNICT)
} 
$[i, i+j]:$ the set $\{i, i+1, \ldots i+j\}$, where $i \geq 0, j \geq 1$.

$\mathcal{S}$ : the variety of semilattices $[x y=y x]$.

$\mathcal{L B}$ : the lattice of varieties of bands.

$\mathcal{L B}_{0}$ : the lattice obtained from $\mathcal{L B}$ by taking away the varieties of rectangular bands.

$\mathcal{L}, \mathcal{J}:$ the Green relations.

Given a function $t: A^{*} \rightarrow A^{*}$, we denote by $\bar{t}$ the function from $A^{*}$ into $A^{*}$ defined by $\bar{t}(w)=\overline{t(\bar{w})}$.

Let $w=u x v$, where $c(w)=c(u x)$ and $c(w) \neq c(u)$. Set

$s(w)=u$ : the longest left cut of $w$ that contains all but one of the variables of $w$.

$\sigma(w)=x$ : the last variable to occur in $w$ in order from the left.

$e(w)=\bar{s}(w)$ : the longest right cut of $w$ that contains all but one of the variables of $w$.

$\varepsilon(w)=\bar{\sigma}(w)$ : the last variable to occur in $w$ in order from the right.

Following Fennemore [3], we define the words $R_{n}, S_{n}$ and $Q_{n}$ for $n \geq 2$, as follows.

$$
\begin{aligned}
& R_{2}=R_{2}\left(x_{1}, x_{2}, x_{3}\right)=x_{3} x_{2} x_{1}, \\
& R_{3}=R_{3}\left(x_{1}, x_{2}, x_{3}\right)=x_{1} x_{2} x_{3}, \\
& Q_{2}=Q_{2}\left(x_{1}, x_{2}, x_{3}\right)=x_{2} x_{3} x_{1}, \\
& Q_{3}=Q_{3}\left(x_{1}, x_{2}, x_{3}\right)=x_{1} x_{2} x_{3} x_{1} x_{3}, \\
& S_{2}=S_{2}\left(x_{1}, x_{2}, x_{3}\right)=x_{3} x_{1} x_{2} x_{1}, \\
& S_{3}=S_{3}\left(x_{1}, x_{2}, x_{3}\right)=x_{1} x_{2} x_{3} x_{1} x_{3} x_{2} x_{3}, \\
& R_{n}=R_{n}\left(x_{1}, \ldots, x_{n}\right)=R_{n-1} x_{n}, \text { for } n=4,6 \ldots \\
& R_{n}=R_{n}\left(x_{1}, \ldots, x_{n}\right)=x_{n} R_{n-1}, \text { for } n=5,7 \ldots \\
& Q_{n}=Q_{n}\left(x_{1}, \ldots, x_{n}\right)=Q_{n-1} x_{n} R_{n}, \text { for } n=4,6 \ldots \\
& Q_{n}=Q_{n}\left(x_{1}, \ldots, x_{n}\right)=R_{n} x_{n} Q_{n-1}, \text { for } n=5,7 \ldots \\
& S_{n}=S_{n}\left(x_{1}, \ldots, x_{n}\right)=S_{n-1} x_{n} R_{n}, \text { for } n=4,6 \ldots \\
& S_{n}=S_{n}\left(x_{1}, \ldots, x_{n}\right)=R_{n} x_{n} S_{n-1}, \text { for } n=5,7 \ldots
\end{aligned}
$$

Set $V_{1}=\mathcal{S}, V_{2}=[x y z=x y], V_{2}^{\prime}=\left[\bar{R}_{2}=\bar{Q}_{2}\right], V_{n}=\left[R_{n}=S_{n}\right], V_{n}^{\prime}=\left[R_{n}=Q_{n}\right]$ for $n \geq 3$, n odd, $V_{n}=\left[\bar{R}_{n}=\bar{S}_{n}\right], V_{n}^{\prime}=\left[\bar{R}_{n}=\bar{Q}_{n}\right]$ for $n \geq 3$, n even. Let $V_{\infty}$ denote the variety of all bands. The lattice $\mathcal{L} \mathcal{B}_{0}$ is represented in Figure 1 .

We call the varieties $V_{1}, V_{\infty}, V_{n}$ and $V_{n}^{\prime}, n \geq 2$, left varieties. The duals of these varieties are called right varieties.

Given a variety of bands $V$, we denote by $V^{\prime}\left[V^{r}\right]$ the minimum of the set of left varieties [right varieties] containing $V$.

Let $S$ be a semigroup. We define the relations $\leq_{C}$ and $<_{L}$ by

$$
\begin{aligned}
& a \leq_{\mathcal{L}} b \Leftrightarrow S^{1} a \subseteq S^{1} b \quad \text { and } \\
& a<_{\mathcal{L}} b \Leftrightarrow S^{\prime} a \subseteq S^{\prime} b \quad \text { but } \quad S^{\prime} a \neq S^{\prime} b \quad(a, b \in S) .
\end{aligned}
$$

Remark 2.1. Let $S$ be a band and let $s, t \in S$ be such that $s \leq_{c} t$. Then $s \mathcal{L} t$ if and only if $s \mathcal{J} t$. Hence, if $u, v \in S$ are such that $c(u)=c(v)$ and $u \leq_{\mathcal{L}} v$, then $u \mathcal{L} v$. 


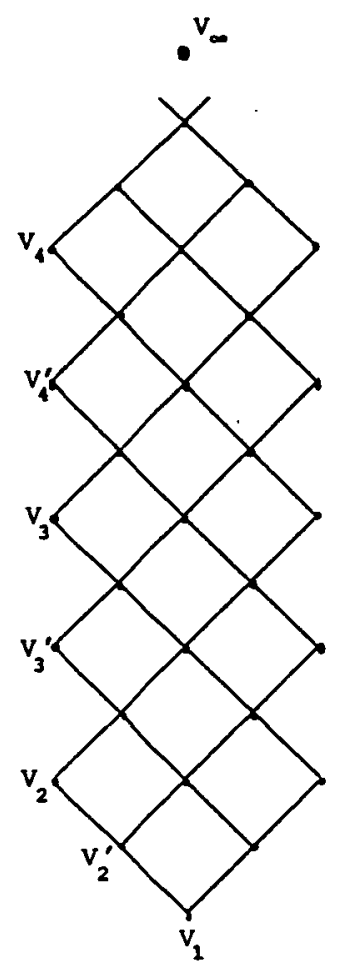

FIGURE 1 The lattice $\mathcal{L} \mathcal{B}_{0}$.

Theorem 2.2. [10] Let us fix a variety of bands $V$. Given a finite subset $B$ of $A$, let $\mathcal{X}_{B}(V)$ denote the $\mathcal{J}$-class of $F_{A}(V)$ consisting of the elements of content $B$. Let $c_{n}(V)$ denote the cardinality of $\mathcal{X}_{B}(V)$ when $|B|$ equals $n$. Then

$$
\begin{aligned}
& c_{k}\left(V_{2}\right)=k !, \\
& c_{k}\left(V_{n}\right)=k^{2} c_{k-1}\left(V_{n}\right) c_{k-1}\left(\bar{V}_{n-1}\right), \quad n \geq 3, \\
& c_{k}\left(V_{n} \vee \bar{V}_{n}\right)=k^{2} c_{k-1}^{2}\left(V_{n}\right), \quad n \geq 2 .
\end{aligned}
$$

\section{The Rhodes expansion}

Let $S$ be a semigroup. A finite sequence $\bar{a}=\left(a_{n}, \ldots, a_{1}\right)$ of elements of $S$ is an $\mathcal{L}$-chain of

$$
a_{n} \leq_{\mathcal{L}} a_{n-1} \leq_{\mathcal{L}} \cdots \leq_{\mathcal{L}} a_{1}
$$


and is a reduced $\mathcal{L}$-chain if

$$
a_{n}<_{\mathcal{L}} a_{n-1}<_{\mathcal{L}} \ldots<_{\mathcal{L}} a_{1} .
$$

The reduction $\operatorname{Red}(\bar{a})$ of $\bar{a}$ is the sequence obtained from $\bar{a}$ by successively deleting the right most element of any pair of $\mathcal{L}$-equivalent elements until no such pairs remain.

Definition 3.1. Let $S$ be a semigroup. The Left Rhodes expansion $\hat{S}^{\mathcal{L}}$ of $S$ is the set of all reduced $\mathcal{L}$-chains with the multiplication

$$
\left(a_{n}<_{\mathcal{L}} \ldots<_{\mathcal{L}} a_{1}\right)\left(b_{m}<_{\mathcal{L}} \ldots<_{\mathcal{L}} b_{1}\right)=\operatorname{Red}\left(a_{n} b_{m} \leq_{\mathcal{L}} \ldots \leq_{\mathcal{L}} a_{1} b_{m} \leq_{\mathcal{L}} b_{m}<_{\mathcal{L}} \ldots<_{\mathcal{L}} b_{1}\right) .
$$

We define a map $\eta_{S}: \hat{S}^{\mathcal{L}} \rightarrow S$ by

$$
\eta_{S}\left(a_{n}<_{\mathcal{L}} \ldots<_{\mathcal{L}} a_{1}\right)=a_{n}
$$

$\eta_{S}$ is a surjective morphism and is called the canonical morphism.

Given a morphism $\varphi: S \rightarrow T$, we define a morphism $\hat{\varphi}^{\mathcal{L}}: \hat{S}^{\mathcal{L}} \rightarrow \hat{T}^{\mathcal{L}}$ by

$$
\hat{\varphi}^{\mathcal{L}}\left(a_{n}<_{\mathcal{L}} \ldots<_{\mathcal{L}} a_{1}\right)=\operatorname{Red}\left(\varphi\left(a_{n}\right) \leq_{\mathcal{L}} \cdots \leq_{\mathcal{L}} \varphi\left(a_{1}\right)\right)
$$

It is easy to check that we get an expansion.

Dually, we can define the right Rhodes expansion $\hat{S}^{\mathcal{R}}$ of $S$.

We refer to [1] for a discussion of the basic properties of the left and right $\mathrm{Rhodes}$ expansions.

We now consider the category $\mathcal{S}_{A}$ of semigroups generated by a given set $A$. Its objects are the pairs $(S, f)$, where $S$ is a semigroup and $f: A \rightarrow S$ is a map such that $f(A)$ generates $S$ (in the classical sense). A morphism from $(S, f)$ into $(T, g)$ is a morphism of semigroups $\varphi: S \rightarrow T$ such that the following diagram is commutative.

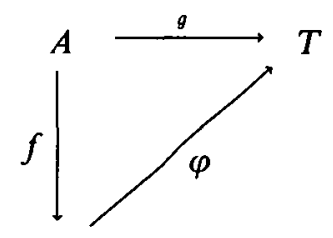

$S$

Definition 3.2. Let $(S, f)$ be an object of $\mathcal{S}_{A}$. The left Rhodes expansion cutdown to generators $A$ of $S$ is the pair $\left(\hat{S}_{A}^{\mathcal{L}}, \hat{f}_{A}^{\mathcal{L}}\right)$, where $\hat{S}_{A}^{\mathcal{L}}$ is the subsemigroup of $\hat{S}^{\mathcal{L}}$ generated by the set $\{(f(a)): a \in A\}$ and $\hat{f}_{A}^{\mathcal{C}}: A \rightarrow \hat{S}_{A}^{\mathcal{C}}$ is defined by

$$
\hat{f}_{A}^{c}(a)=(f(a)) \quad(a \in A)
$$


The canonical morphism $\eta_{S, A}: \hat{S}_{A}^{\mathcal{L}} \rightarrow S$ is defined simply by

$$
\eta_{S, A}=\left.\eta_{S}\right|_{\hat{s}_{A}^{c}}
$$

Finally, if $\varphi$ is a morphism from $(S, f)$ into $(T, g)$, we define $\hat{\varphi}_{A}^{\mathcal{L}}$ from $\left(\hat{S}_{A}^{\mathcal{L}}, \hat{f}_{A}^{\mathcal{L}}\right)$ into $\left(\hat{T}_{A}^{\mathcal{L}}, \hat{g}_{A}^{\mathcal{L}}\right)$ by

$$
\hat{\varphi}_{A}^{\mathcal{L}}=\left.\hat{\varphi}^{\mathcal{L}}\right|_{\hat{S}_{A}^{c}}
$$

It is easy to check that we still get an expansion.

We refer again to [1] for a discussion of the basic properties of the left and right Rhodes expansion cutdown to generators.

From now on we work with the left Rhodes expansions cutdown to generators and we omit the word "left".

Theorem 3.3. [Tilson, 11] Let $S$ be a semigroup. Then the Rhodes expansion cutdown to $A$ of $\hat{S}_{A}^{\mathcal{L}}$ is isomorphic to $\hat{S}_{A}^{\mathcal{L}}$.

Fact 3.4. If $(S, f)$ and $(T, g)$ are objects of $S_{A}$, there is at most one morphism from $(S, f)$ to $(T, g)$. Consequently, if $\varphi:(S, f) \rightarrow(T, g)$ and $\psi:(T, g) \rightarrow(S, f)$ are morphisms, then $(S, f)$ and $(T, g)$ are isomorphic.

Fact 3.5. [1] If $S$ is a band, then $\hat{S}_{A}^{\mathcal{L}}$ is also a band.

Lemma 3.6. Let $S$ be a semigroup and let $x, y \in \hat{S}^{\mathcal{L}}$. Then

$$
x y=y \Leftrightarrow \eta_{s}(x y)=\eta_{s}(y)
$$

Proof. Let $x=\left(x_{n}<_{\mathcal{L}} \cdots<_{\mathcal{L}} x_{1}\right), y=\left(y_{m}<_{\mathcal{L}} \cdots<_{\mathcal{L}} y_{1}\right), x_{1}, \ldots, x_{n}, y_{1}, \ldots y_{m} \in S$, be such that $\eta_{s}(x y)=\eta_{s}(y)$. This means that

$$
\eta_{S}\left(\operatorname{Red}\left(x_{n} y_{m} \leq_{\mathcal{L}} \cdots \leq_{\mathcal{L}} x_{1} y_{m} \leq_{\mathcal{L}} y_{m}<_{\mathcal{L}} \cdots<_{\mathcal{L}} y_{1}\right)\right)=\eta_{S}\left(y_{m}<_{\mathcal{L}} \cdots<_{\mathcal{L}} y_{1}\right)
$$

that is,

$$
\eta_{s}\left(\operatorname{Red}\left(x_{n} y_{m} \leq_{c} \cdots \leq_{c} x_{1} y_{m} \leq_{c} y_{m}<_{c} \cdots<_{c} y_{1}\right)\right)=y_{m} .
$$

By the definitions of Red and $\eta_{s}$ we get

$$
\operatorname{Red}\left(x_{n} y_{m} \leq_{\mathcal{C}} \cdots \leq_{\mathcal{L}} x_{1} y_{m} \leq_{\mathcal{L}} y_{m}<_{\mathcal{L}} \cdots<_{\mathcal{L}} y_{1}\right)=\left(y_{m}<_{\mathcal{L}} \cdots<_{\mathcal{L}} y_{1}\right)
$$

that is, $x y=y$.

The converse is trivial. 


\section{The Rhodes expansions of the free objects}

If $S$ is a rectangular band, then $\hat{S}_{A}^{\mathcal{L}}$ is isomorphic to $S$. This comes from the fact that all the sequences $\left(s_{n}<_{\mathcal{L}} \cdots<_{\mathcal{L}} s_{1}\right)$ have length one, since, if $s, t \in S$ and $s \leq_{\mathcal{L}} t$, then $s \mathcal{L}$.

From now on we work on $\mathcal{L B}_{0}$.

We start with $V=\mathcal{S}$ and show that if $S=F_{A}(V)$, then $\hat{S}_{A}^{\mathcal{C}} \simeq F_{A}\left(\bar{V}_{2}\right)$. In order to show this, we will show that:

(1) If $S \in S$, then $\hat{S}^{\mathcal{L}} \in \bar{V}_{2}$.

(2) If $S=F_{A}(V)$, then $\hat{S}_{A}^{c}=\left\{\left(s_{k}<_{\mathcal{L}} \cdots<_{\mathcal{L}} s_{1}\right): k \geq 1, s_{i} \in A^{+},\left|c\left(s_{i}\right)\right|=i, i \in[1, k]\right\}$ and $\left|\hat{S}_{A}^{\mathcal{L}}\right|=\left|F_{A}\left(\bar{V}_{2}\right)\right|$.

Proof of (1). Let $s, t \in \hat{S}^{\mathcal{L}}, s=\left(s_{k}<_{\mathcal{L}} \cdots<_{\mathcal{L}} s_{1}\right), t=\left(i_{l}<_{\mathcal{L}} \cdots<_{\mathcal{L}} i_{1}\right)$, where $k, l \geq 1$, $s_{i}, i_{j} \in A^{+},\left|c\left(s_{i}\right)\right|=i,\left|c\left(i_{j}\right)\right|=j, i \in[1, k], j \in[1, l]$. Then

$$
\begin{aligned}
\text { sts } & =\operatorname{Red}\left(s_{k} i_{1} \leq_{\mathcal{L}} \cdots \leq_{\mathcal{L}} s_{1} i_{l}<_{\mathcal{L}} i_{\mathcal{L}}<_{\mathcal{L}} \cdots<_{\mathcal{L}} i_{1}\right) \cdot\left(s_{k}<_{\mathcal{L}} \cdots<_{\mathcal{L}} s_{1}\right) \\
& =\operatorname{Red}\left(s_{k} i_{l} s_{k} \leq_{\mathcal{L}} \cdots \leq_{\mathcal{L}} s_{1} i_{l} s_{k} \leq_{\mathcal{L}} i_{l} s_{k} \leq_{\mathcal{L}} \cdots \leq_{\mathcal{L}} i_{1} s_{k} \leq_{\mathcal{L}} s_{k}<_{\mathcal{L}} \cdots<_{\mathcal{L}} s_{1}\right) \\
& =\operatorname{Red}\left(i_{l} s_{k} \leq_{\mathcal{L}} \cdots \leq_{\mathcal{L}} i_{1} s_{k} \leq_{\mathcal{L}} s_{k}<_{\mathcal{L}} \cdots<_{\mathcal{L}} s_{1}\right) \\
& =t s .
\end{aligned}
$$

Proof of (2). Let $X=\left\{\left(s_{k}<_{\mathcal{L}} \cdots<_{\mathcal{L}} s_{1}\right): k \geq 1, s_{i} \in A^{+},\left|c\left(s_{i}\right)\right|=i, i \in[1, k]\right\}$.

Let $s \in \hat{S}_{A}^{\mathcal{L}}$. If $s=(a), a \in A$, then $s \in X$. Suppose that any product $\left(a_{n}\right) \ldots\left(a_{1}\right)$, $n \geq 1, a_{i} \in A, i \in[1, n]$ is in $X$. Let $a_{n+1} \in A$. Then

$$
\begin{aligned}
\left(a_{n+1}\right) \ldots\left(a_{1}\right) & =\left(a_{n+1}\right)\left[\left(a_{n}\right) \ldots\left(a_{1}\right)\right] \\
& =\left(a_{n+1}\right)\left(s_{k}<_{\mathcal{L}} \cdots<_{\mathcal{L}} s_{1}\right)
\end{aligned}
$$

by the induction hypothesis, where $s_{i} \in A^{+},\left|c\left(s_{i}\right)\right|=i, i \in[1, k]$. Hence

$$
\left(a_{n+1}\right) \ldots\left(a_{1}\right)=\operatorname{Red}\left(a_{n+1} s_{k} \leq_{\mathcal{L}} s_{k}<_{\mathcal{L}} \cdots<_{\mathcal{L}} s_{1}\right) .
$$

If $a_{n+1} \notin c\left(s_{k}\right)$, then $a_{n+1} s_{k}<_{\mathcal{L}} s_{k}$ and

$$
\left(a_{n+1}\right) \ldots\left(a_{1}\right)=\left(a_{n+1} s_{k}<_{\mathcal{L}} s_{k}<_{\mathcal{L}} \cdots<_{\mathcal{L}} s_{1}\right),
$$

with $\left|c\left(a_{n+1} s_{k}\right)\right|=k+1$.

If $a_{n+1} \in c\left(s_{k}\right)$, then $a_{n+1} s_{k} \mathcal{L} s_{k}$ and

$$
\left(a_{n+1}\right) \ldots\left(a_{1}\right)=\left(a_{n+1} s_{k}<_{\mathcal{L}} s_{n-1}<_{\mathcal{L}} \cdots<_{\mathcal{L}} s_{1}\right),
$$

where $\left|c\left(a_{n+1} s_{k}\right)\right|=k$.

Thus $\hat{S}_{A}^{\mathcal{L}} \subseteq X$.

Conversely, let $s=\left(s_{k}<_{\mathcal{L}} \cdots<_{\mathcal{L}} s_{1}\right) \in X$ and let $\left\{a_{i}\right\}=c\left(s_{i}\right) \backslash c\left(s_{i-1}\right), i \in[1, k]$. In $S$, $s_{i}=a_{i} s_{i-1}, i \in[1, k]$ (since $\left.c\left(s_{i}\right)=c\left(a_{i} s_{i-1}\right)\right)$. It is then a mere routine to check that $s=\left(a_{k}\right) \ldots\left(a_{1}\right)$. Thus, $s \in \hat{S}_{A}^{\mathcal{L}}$. 
Finally, if $|A|=N$, we have

$$
\begin{aligned}
\left|\hat{S}_{A}^{c}\right| & \left.=\|\left(s_{k}<_{\mathcal{L}} \cdots<_{\mathcal{L}} s_{1}\right): k \geq 1, s_{i} \in A^{+},\left|c\left(s_{i}\right)\right|=i, i \in[1, k]\right\} \mid \\
& =\sum_{k=1}^{N}\left|\left\{\left(s_{k}, \ldots, s_{1}\right): s_{i} \in A^{+}, c\left(s_{i}\right) \supseteq c\left(s_{i-1}\right),\left|c\left(s_{i}\right)\right|=i, i \in[1, k]\right\}\right| \\
& =\sum_{k=1}^{N} N(N-1) \ldots(N-k+1) \\
& =\left|F_{A}\left(\bar{V}_{2}\right)\right| \quad \text { (See Theorem 2.2.) }
\end{aligned}
$$

We now state the main theorem. As mentioned in the introduction, this is a special case of a result due to Reilly [8].

Theorem 4.1. If $V \in \mathcal{L} \mathcal{B}_{0}$ and $S=F_{A}(V)$, then $\hat{S}_{A}^{c} \simeq F_{A}\left(V^{r}\right)$.

The next statement shows that it suffices to prove the theorem for the left varieties of $\mathcal{L B}_{0}$. Moreover, since the remaining cases can be proved similarly, we shall only consider the cases $V=V_{2}$ or $V=V_{k}, k$ odd, $k \geq 3$.

Proposition 4.2. Let $S, T, U \in S_{A}$ and let $\varphi: S \rightarrow T, \psi: T \rightarrow U$ be morphisms. Then

$$
\hat{U}_{A}^{\mathcal{L}} \simeq S \Rightarrow \hat{T}_{A}^{\mathcal{L}} \simeq S .
$$

Proof. Let $S, T, U \in S_{A}$ and let $\varphi: S \rightarrow T, \psi: T \rightarrow U$ be morphisms. Suppose that $U_{A}^{C} \simeq S$ and let $\chi$ be an isomorphism from $\hat{U}_{A}^{c}$ into $S$.

By Theorem 3.3, $\hat{S}_{A}^{c} \simeq S$ and by Fact 3.4, the natural morphism $\eta_{S, A}: \hat{S}_{A}^{c} \rightarrow S$ is an isomorphism. Let $\eta_{S, A}^{-1}$ be its inverse. Let $\hat{\varphi}_{A}^{C}: \hat{S}_{A}^{C} \rightarrow \hat{T}_{A}^{C}$ and $\hat{\psi}_{A}^{C}: \hat{T}_{A}^{C} \rightarrow \hat{U}_{A}^{c}$ be the morphisms determined by $\varphi$ and $\psi$, respectively. Then $\hat{\varphi}_{A}^{C} \circ \eta_{S, A}^{-1} \circ \chi \circ \hat{\psi}_{A}^{C}$ is a morphism from $\hat{T}_{A}^{\mathcal{C}}$ into itself and so it is the identity morphism. Hence $\chi \circ \hat{\psi}_{A}^{c}$ is an isomorphism from $\hat{T}_{A}^{\mathcal{C}}$ into $S$.

We now state a series of lemmas, whose proofs we shall defer until we prove Theorem 4.1.

Lemma 4.3. If $V$ is a left variety and $S \in V$, then $\hat{S}^{\mathcal{L}} \in V^{r}$.

Lemma 4.4. Let $u, v \in A^{+}$be such that $c(u) \supseteq c(v),|c(u)|=|c(v)|+1$.

(i) If $V=V_{2}$, then $u<_{C} v$ in $F_{A}(V)$.

(ii) If $V=V_{k}$ odd, $k \geq 3$, then

$$
i_{k}(u) \leq_{\mathcal{L}} i_{k}(v) \Leftrightarrow \bar{i}_{k-1}(e(u)) \mathcal{L} \bar{i}_{k-1}(v) .
$$

Proposition 4.5. Let $S=F_{A}(V)$. 
(i) If $V=V_{2}$, then

$$
\hat{S}_{A}^{\mathcal{L}}=\left\{\left(s_{n}<_{\mathcal{L}} \cdots<_{\mathcal{L}} s_{1}\right): n \geq 1, s_{i} \in A^{+},\left|c\left(s_{i}\right)\right|=i, i \in[1, n]\right\} .
$$

(ii) If $V=V_{k}, k$ odd, $k \geq 3$, then

$$
\hat{S}_{A}^{\mathcal{L}}=\left\{\left(s_{n}<_{\mathcal{L}} \cdots<_{\mathcal{L}} s_{1}\right): n \geq 1, s_{i} \in A^{+},\left|\mathcal{C}\left(s_{i}\right)\right|=i, \bar{i}_{k-1}\left(e\left(s_{i}\right)\right)=\bar{i}_{k-1}\left(s_{i-1}\right), i \in[1, n]\right\} .
$$

Lemma 4.6. Let $n \geq 1$ and let $u \in A^{+}$be such that $|c(u)|=n$.

(i) $V=V_{2}$, then

$$
\left|\left\{\left(u \leq_{\mathcal{L}} s_{n-1} \leq_{\mathcal{C}} \cdots \leq_{\mathcal{L}} s_{1}\right): s_{i} \in A^{+},\left|c\left(s_{i}\right)\right|=i, i \in[1, n-1]\right\}\right|=\frac{c_{n}\left(\bar{V}_{3}\right)}{c_{n}\left(V_{2}\right)} .
$$

(ii) If $V=V_{k}, k$ odd, $k \geq 3$, then

$$
\begin{aligned}
& \|\left(u \leq_{\mathcal{L}} s_{n-1} \leq_{\mathcal{L}} \cdots \leq_{\mathcal{L}} s_{1}\right): s_{i} \in A^{+},\left|c\left(s_{i}\right)\right|=i, \bar{i}_{k-1}(e(u))=\bar{i}_{k-1}\left(s_{n-1}\right), \\
& \left.\quad \bar{i}_{k-1}\left(e\left(s_{i}\right)\right)=\bar{i}_{k-1}\left(s_{i-1}\right), i \in[1, n-1]\right\} \mid \\
& \quad=\frac{c_{n}\left(\bar{V}_{k+1}\right)}{c_{n}\left(V_{k}\right)} .
\end{aligned}
$$

Lemma 4.7. Let $n \leq 1$ and let $A_{n} \subseteq A$ be such that $\left|A_{n}\right|=n$. Let $S=F_{A}\left(V_{k}\right), k=2$ or $k$ odd, $k \geq 3$. Then

$$
\left|\left\{s \in \hat{S}_{A}^{\mathcal{L}}: c\left(\eta_{S, A}(s)\right)=A_{n}\right\}\right|=c_{n}\left(\bar{V}_{k+1}\right)
$$

Proof of Theorem 4.1. Let $S=F_{A}(V)$. If $V=V_{\infty}$, by Fact $3.5, \hat{S}_{A}^{\mathcal{L}} \in V_{\infty}$. Consequently, there is a morphism $\psi: S \rightarrow \hat{S}_{A}^{L}$. Since $\eta_{S_{\mathcal{A}} \mathcal{C}^{\infty}}$ is a morphism from $\hat{S}_{A}^{\mathcal{L}}$ into $S$, Fact 3.4 yields that $\varphi, \eta_{S, A}$ are isomorphisms and so $\hat{S}_{A}^{\mathcal{L}} \simeq S$.

Let $S=F_{A}\left(V_{k}\right), k \geq 2$. By Lemma 4.3 there is a surjective morphism $\varphi: F_{A}\left(\bar{V}_{k+1}\right) \rightarrow \hat{S}_{A}^{\mathcal{L}}$ such that the following diagram is commutative.

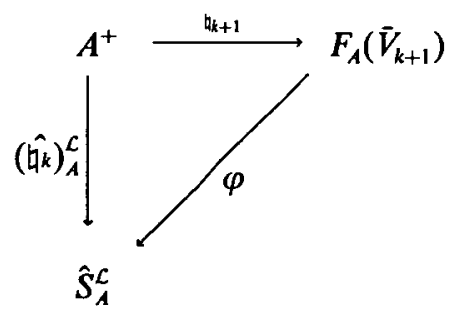

where $\natural_{k}$ denotes the canonical epimorphism from $A^{+}$into $F_{A}\left(V_{k}\right)$. 
Also the following diagram is commutative.

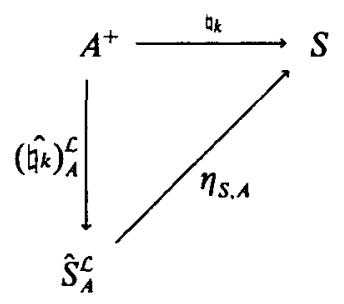

We now show that $\varphi$ is injective. It is enough to show that the restriction of $\varphi$ to the $\mathcal{J}$-classes of $F_{A}\left(\bar{V}_{k+1}\right)$ is injective. Indeed, if $t$ and $t^{\prime} \in F_{A}\left(\bar{V}_{k+1}\right)$ are such that $\varphi(t)=\varphi\left(t^{\prime}\right)$, then there are $u, v \in A^{+}$such that $t=a_{k+1}(u), t^{\prime}=a_{k+1}(v)$ and so we get

$$
\begin{aligned}
\varphi(t)=\varphi\left(t^{\prime}\right) & \Rightarrow \varphi \natural_{k+1}(u)=\varphi \natural_{k+1}(v) \\
& \Rightarrow\left(\hat{\natural_{k}}\right)_{A}^{\mathcal{L}}(u)=\left(\hat{\natural_{k}}\right)_{A}^{\mathcal{L}}(v) \quad \text { by }(4.1 .1) \\
& \Rightarrow \eta_{S \cdot A}\left(\hat{\natural_{k}}\right)_{A}^{\mathcal{L}}(u)=\eta_{S \cdot A}\left(\hat{\varphi_{k}}\right)_{A}^{\mathcal{L}}(v) \\
& \Rightarrow \natural_{k}(u)=\bigsqcup_{k}(v) \quad \text { by }(4.1 .2) \\
& \Rightarrow c(u)=c(v)
\end{aligned}
$$

and therefore $t$ and $t^{\prime}$ are $\mathcal{J}$-related in $F_{A}\left(\bar{V}_{k+1}\right)$.

Let $A_{n} \subseteq A$. We have

$$
\varphi\left(\left\{t \in F_{A}\left(\bar{V}_{k+1}\right): c(t)=A_{n}\right\}\right)=\left\{\varphi(t) \in \hat{S}_{A}^{\mathcal{L}}: c(t)=A_{n}\right\}
$$

But if $t=h_{k+1}(u), u \in A^{+}$we get

$$
\begin{aligned}
c(t) & =c\left(\natural_{k+1}(u)\right) \\
& =c\left(\natural_{k}(u)\right) \\
& =c\left(\eta_{S, A}\left(\hat{\natural_{k}}\right)_{A}^{\mathcal{L}}(u)\right) \quad \text { by }(4.1 .2) \\
& =c\left(\eta_{S, A} \varphi \natural_{k+1}(u)\right) \quad \text { by }(4.1 .1) \\
& =c\left(\eta_{S, A}(\varphi(t))\right) .
\end{aligned}
$$

Hence

$$
\begin{aligned}
\varphi\left(\left\{t \in F_{A}\left(\bar{V}_{k+1}\right): c(t)=A_{n}\right\}\right) & =\left\{\varphi(t) \in \hat{S}_{A}^{\mathcal{L}}: c\left(\eta_{S . A}(\varphi(t))\right)=A_{n}\right\} \\
& =\left\{s \in \hat{S}_{A}^{\mathcal{L}}: c\left(\eta_{S, A}(s)\right)=A_{n}\right\}
\end{aligned}
$$

and the injectivity of $\varphi$ follows from the fact that 


$$
\begin{aligned}
& \left|\varphi\left(\left\{t \in F_{A}\left(\bar{V}_{k+1}\right): c(t)=A_{n}\right\}\right)\right| \\
& \quad=\left|\left\{s \in \hat{S}_{A}^{\mathcal{L}}: c\left(\eta_{s, A}(s)\right)=A_{n}\right\}\right| \\
& \quad=c_{n}\left(\bar{V}_{k+1}\right) \quad \text { by Lemma } 4.7 \\
& \quad=\left|\left\{t \in F_{A}\left(\bar{V}_{k+1}\right): c(t)=A_{n}\right\}\right| .
\end{aligned}
$$

We now prove 4.3-4.7.

Proof of Lemma 4.3. The proof is based on Lemma 3.6. The case of $\mathcal{S}$ will be omitted since it was already treated.

If $S \in V_{\infty}$ the result is an immediate consequence of Fact 3.5.

Let $S \in V_{2}$ and let $s_{1}, s_{2}, s_{3} \in \hat{S}^{\mathcal{C}}$. Then

$$
\begin{aligned}
\eta_{s}\left(s_{1} s_{2} s_{1} s_{3} s_{1} s_{2} s_{3}\right) & =\eta_{s}\left(s_{1}\right) \eta_{s}\left(s_{2}\right) \eta_{s}\left(s_{1}\right) \eta_{s}\left(s_{3}\right) \eta_{s}\left(s_{1}\right) \eta_{s}\left(s_{2}\right) \eta_{s}\left(s_{3}\right) \\
& =\eta_{s}\left(s_{1}\right) \eta_{s}\left(s_{2}\right) \eta_{s}\left(s_{3}\right) \eta_{s}\left(s_{1}\right) \eta_{s}\left(s_{2}\right) \eta_{s}\left(s_{3}\right) \\
& =\eta_{s}\left(s_{1}\right) \eta_{s}\left(s_{2}\right) \eta_{s}\left(s_{3}\right) \\
& =\eta_{s}\left(s_{1} s_{2} s_{3}\right)
\end{aligned}
$$

Hence Lemma 3.6 yields that $s_{1} s_{2} s_{3}=s_{1} s_{2} s_{1} s_{3} s_{1} s_{2} s_{3}$, thus $\hat{S}^{\mathcal{L}} \in \bar{V}_{3}$, as required.

Now let $S \in V_{k}, k$ odd, $k \geq 3$. By definition $R_{k+1}=R_{k} x_{k+1}$ and $S_{k+1}=S_{k} x_{k+1} R_{k+1}$. We notice first that if $S_{k}=S_{k}\left(x_{1}, \ldots, x_{k}\right)$, then

$$
\begin{aligned}
\eta_{S}\left(S_{k}\right) & =S_{k}\left(\eta_{s}\left(x_{1}\right), \ldots, \eta_{s}\left(x_{k}\right)\right) \\
& =R_{k}\left(\eta_{S}\left(x_{1}\right), \ldots, \eta_{s}\left(x_{k}\right)\right) \\
& =\eta_{s}\left(R_{k}\right) .
\end{aligned}
$$

Hence,

$$
\begin{aligned}
\eta_{s}\left(S_{k} x_{k+1} R_{k+1}\right) & =\eta_{s}\left(S_{k}\right) \eta_{s}\left(x_{k+1}\right) \eta_{s}\left(R_{k+1}\right) \\
& =\eta_{s}\left(R_{k}\right) \eta_{s}\left(x_{k+1}\right) \eta_{s}\left(R_{k+1}\right) \\
& =\eta_{s}\left(R_{k} x_{k+1}\right) \eta_{s}\left(R_{k+1}\right) \\
& =\eta_{s}\left(R_{k+1}\right) \eta_{s}\left(R_{k+1}\right) \\
& =\eta_{s}\left(R_{k+1}\right)
\end{aligned}
$$

Thus Lemma 3.6 yields that $S_{k+1}=R_{k+1}$ and so $\hat{S}^{\mathcal{L}} \in \bar{V}_{k+1}$, as required.

Proof of Lemma 4.4. Let $u, v \in A^{+}$be such that $c(u) \supseteq c(v),|c(u)|=|c(v)|+1$.

(i) Let $V=V_{2}$. Then

$$
u \leq_{\mathcal{L}} v \Leftrightarrow i_{2}(u v)=i_{2}(u)
$$


But $c(u) \supseteq c(v)$ implies $c(u v)=c(u), i_{2}(u v)=i_{2}(u)$. Hence $u \leq_{\complement} v$. If $v \leq_{\complement} u$, we would get $c(v u)=c(v)$ and this contradicts the fact that $c(u) \neq c(v)$. Therefore $u<_{L} v$.

(ii) Let $V=V_{k}, k$ odd, $k \geq 3$. Then

$$
\begin{aligned}
u \leq_{\mathcal{L}} v & \Leftrightarrow i_{k}(u v)=i_{k}(u) \\
& \Leftrightarrow\left\{\begin{array}{l}
i_{k}(s(u v))=i_{k}(s(u)) \\
\sigma(u v)=\sigma(u) \\
\varepsilon(u v)=\varepsilon(u) \\
\bar{i}_{k-1}(e(u v))=\bar{i}_{k-1}(e(u))
\end{array}\right. \\
& \Leftrightarrow \bar{i}_{k-1}(e(u v))=\bar{i}_{k-1}(e(u)), \quad \text { since } c(u) \supseteq c(v) \\
& \Leftrightarrow \bar{i}_{k-1}(e(u) v)=\bar{i}_{k-1}(e(u)), \quad \text { since } c(u) \neq c(v) \\
& \Leftrightarrow \bar{i}_{k-1}(e(u)) \bar{i}_{k-1}(v)=\bar{i}_{k-1}(e(u)) \\
& \Leftrightarrow \bar{i}_{k-1}(e(u)) \leq \bar{i}_{k-1}(v) \\
& \Leftrightarrow \bar{i}_{k-1}(e(u)) \mathcal{L} \bar{i}_{k-1}(v),
\end{aligned}
$$

since $c(e(u))=c(v)$. (See Remark 2.1.)

Proof of Proposition 4.5. (i) Let $V=V_{2}$ and let

$$
X=\left\{\left(s_{n}<_{\mathcal{L}} \cdots<_{\mathcal{L}} s_{1}\right): n \geq 1, s_{i} \in A^{+},\left|c\left(s_{i}\right)\right|=i, i \in[1, n]\right\} .
$$

The proof of the inclusion $\hat{S}_{A}^{\mathcal{L}} \subseteq X$ is analogous with the proof made for $V=\mathcal{S}$. We now prove the converse inclusion.

Let $s \in X$.

If $s=\left(s_{1}\right),\left|c\left(s_{1}\right)\right|=1$, then $s_{1} \in A$ and $s \in \hat{S}_{A}^{\mathcal{L}}$.

Let $n \geq 1$ and suppose that all sequences $\left(s_{n}<_{\mathcal{L}} \cdots<_{\mathcal{L}} s_{1}\right)$ of $X$ are in $\hat{S}_{A}^{\mathcal{L}}$. Let $s=\left(s_{n+1}<_{\mathcal{L}} \cdots<_{\mathcal{L}} s_{1}\right) \in X$ and let $c\left(s_{n+1}\right) \backslash c\left(s_{n}\right)=\left\{a_{n+1}\right\}$. It is easy to see that

$$
i_{2}\left(s_{n+1}\right)=i_{2}\left(s\left(s_{n+1}\right) \sigma\left(s_{n+1}\right) a_{n+1} s_{n}\right)
$$

Now let $s\left(s_{n+1}\right)=b_{1} \ldots b_{r}, b_{i} \in A, i \in[1, r]$. We will see that

$$
s=\left(b_{1}\right) \ldots\left(b_{r}\right)\left(\sigma\left(s_{n+1}\right)\right)\left(a_{n+1}\right)\left(s_{n}<_{\mathcal{C}} \cdots<_{\mathcal{L}} s_{1}\right) .
$$

Indeed,

$$
\begin{aligned}
\left(b_{1}\right) & \ldots\left(b_{r}\right)\left(\sigma\left(s_{n+1}\right)\right)\left(a_{n+1}\right)\left(s_{n}<_{\mathcal{C}} \cdots<_{\mathcal{C}} s_{1}\right) \\
& =\operatorname{Red}\left(b_{1} \ldots b_{r} \sigma\left(s_{n+1}\right) s_{n+1} s_{n} \leq_{\mathcal{C}} b_{2} \ldots b_{r} \sigma\left(s_{n+1}\right) a_{n+1} s_{n}<_{\mathcal{C}} s_{n}<_{\mathcal{C}} \cdots<_{\mathcal{C}} s_{1}\right) \\
& =\left(b_{1} \ldots b_{r} \sigma\left(s_{n+1}\right) a_{n+1} s_{n}<_{C} s_{n}<_{\mathcal{L}} \cdots<_{\mathcal{C}} s_{1}\right)
\end{aligned}
$$

since $c\left(c_{1} \ldots b_{r} \sigma\left(s_{n+1}\right) a_{n+1} s_{n}\right)=c\left(a_{n+1} s_{n}\right)$. Thus 


$$
\begin{aligned}
\left(b_{1}\right) \ldots\left(b_{r}\right)\left(\sigma\left(s_{n+1}\right)\right)\left(a_{n+1}\right)\left(s_{n}<_{\mathcal{L}} \ldots<_{\mathcal{L}} s_{1}\right) & =\left(s\left(s_{n+1}\right) \sigma\left(s_{n+1}\right) a_{n+1} s_{n}<_{\mathcal{L}} s_{n}<_{\mathcal{L}} \ldots<_{\mathcal{L}} s_{1}\right) \\
& =\left(s_{n+1}<_{\mathcal{L}} s_{n}<_{\mathcal{L}} \cdots<_{\mathcal{L}} s_{1}\right) \text { by }(4.5 .1) .
\end{aligned}
$$

Hence the inclusion hypothesis yields that $s \in \hat{S}_{A}^{\mathcal{L}}$.

(ii) Let $V=V_{k}, k$ odd, $k \geq 3$. Let

$$
Y=\left\{\left(s_{n}<_{C} \cdots<_{C} s_{1}\right): n \geq 1, s_{i} \in A^{+},\left|c\left(s_{i}\right)\right|=i, \bar{i}_{k-1}\left(e\left(s_{i}\right)\right)=\bar{i}_{k-1}\left(s_{i-1}\right), i \in[1, n]\right\} .
$$

We prove first the inclusion $\hat{S}_{A}^{\mathcal{L}} \subseteq Y$.

If $a \in A$, then $(a) \in Y$. Suppose that $n \geq 1$ and that any product $\left(a_{n}\right) \ldots\left(a_{1}\right), a_{i} \in A$, $i \in[1, n]$, is in $Y$. Let $s=\left(a_{n+1}\right)\left(a_{n}\right) \ldots\left(a_{1}\right), a_{i} \in A, i \in[1, n+1]$. Then

$$
\begin{aligned}
s & =\left(a_{n+1}\right)\left[\left(a_{n}\right) \ldots\left(a_{1}\right)\right] \\
& =\left(a_{n+1}\right)\left(s_{k}<_{\mathcal{L}} \cdots<_{\mathcal{L}} s_{1}\right)
\end{aligned}
$$

by the induction hypothesis, where $k \geq 1, s_{i} \in A^{+},\left|c\left(s_{i}\right)\right|=i, \bar{i}_{k-1}\left(e\left(s_{i}\right)\right)=\bar{i}_{k-1}\left(s_{i-1}\right), i \in[1, k]$. Hence

$$
s=\operatorname{Red}\left(a_{n+1} s_{k} \leq_{\mathcal{L}} s_{k}<_{\mathcal{L}} \cdots<_{\mathcal{L}} s_{1}\right)
$$

If $a_{n+1} \in c\left(s_{k}\right)$, then $a_{n+1} s_{k} \mathcal{L} s_{k}$ (see Remark 2.1) and

$$
s=\left(a_{n+1} s_{k}<_{\mathcal{L}} s_{k-1}<_{\mathcal{L}} s_{k}<_{\mathcal{L}} \cdots<_{\mathcal{L}} s_{1}\right)
$$

where $\left|c\left(a_{n+1} s_{k}\right)\right|=k$. Moreover in this case

$$
\begin{aligned}
\bar{i}_{k-1}\left(e\left(a_{n+1} s_{k}\right)\right) & =\bar{i}_{k-1}\left(e\left(s_{k}\right)\right) \\
& =\bar{i}_{k-1}\left(s_{k-1}\right), \quad \text { by the induction hypothesis }
\end{aligned}
$$

and so $s \in Y$.

If $a_{n+1} \notin c\left(s_{k}\right)$ then $a_{n+1} s_{k}<_{\mathcal{L}} s_{k}$ and

$$
s=\left(a_{n+1} s_{k}<_{L} s_{k}<_{L} \cdots<_{L} s_{1}\right)
$$

where $\left|c\left(c_{n+1} s_{k}\right)\right|=k+1$. Moreover

$$
\bar{i}_{k-1}\left(e\left(a_{n+1} s_{k}\right)\right)=\bar{i}_{k-1}\left(s_{k}\right)
$$

since in this case $\varepsilon\left(a_{n+1} s_{k}\right)=a_{n+1}$ and $e\left(a_{n+1} s_{k}\right)=s_{k}$.

Hence $\hat{S}_{A}^{\mathcal{L}} \subseteq Y$.

Conversely, let $s \in Y$. If $s=\left(s_{1}\right)$, with $\left|c\left(s_{1}\right)\right|=1$, then $s_{1} \in A$ and $s \in \hat{S}_{A}^{\mathcal{L}}$. Let $n \geq 1$ and suppose that all sequences $\left(s_{n}<_{C} \cdots<_{C} s_{1}\right)$ of $Y$ are in $\hat{S}_{A}^{c}$. Let $s=\left(s_{n+1}<_{\mathcal{L}} s_{n}<_{\mathcal{L}} \cdots<_{\mathcal{L}} s_{1}\right) \in Y$. 


\section{ON RHODES EXPANSIONŚ OF BANDS}

Since $\bar{i}_{k-1}\left(e\left(s_{n+1}\right)\right)=\bar{i}_{k-1}\left(s_{n}\right)$, then $c\left(e\left(s_{n+1}\right)\right)=c\left(s_{n}\right)$ and we deduce that

$$
\left\{\varepsilon\left(s_{n+1}\right)\right\}=c\left(s_{n+1}\right) \backslash c\left(s_{n}\right) .
$$

Again, it is easy $t$ check that

$$
i_{k}\left(s_{n+1}\right)=i_{k}\left(s\left(s_{n+1}\right) \sigma\left(s_{n+1}\right) \varepsilon\left(s_{n+1}\right) s_{n}\right) \text {. }
$$

Now, let $s\left(s_{n+1}\right)=b_{1} \ldots b_{r}, b_{i} \in A, i \in[1, r]$. We show that

$$
s=\left(b_{1}\right) \ldots\left(b_{r}\right)\left(\sigma\left(s_{n+1}\right)\right)\left(\varepsilon\left(s_{n+1}\right)\right)\left(s_{n}<_{c} \cdots<_{c} s_{1}\right) .
$$

Indeed,

$$
\begin{aligned}
\left(b_{1}\right) \ldots & \left(b_{r}\right)\left(\sigma\left(s_{n+1}\right)\right)\left(\varepsilon\left(s_{n+1}\right)\right)\left(s_{n}<_{\mathcal{L}} \cdots<_{\mathcal{L}} s_{1}\right) \\
= & \operatorname{Red}\left(b_{1} \ldots b_{r} \sigma\left(s_{n+1}\right) \varepsilon\left(s_{n+1}\right) s_{n} \leq_{\mathcal{L}} b_{2} \ldots b_{r} \sigma\left(s_{n+1}\right) \varepsilon\left(s_{n+1}\right) s_{n} \leq_{\mathcal{L}} \cdots \leq_{\mathcal{L}}\right. \\
& \left.\sigma\left(s_{n+1}\right) \varepsilon\left(s_{n+1}\right) s_{n} \leq_{\mathcal{L}} \varepsilon\left(s_{n+1}\right) s_{n}<_{\mathcal{L}} s_{n}<_{\mathcal{L}} \cdots<_{\mathcal{L}} s_{1}\right) \\
= & \left(b_{1} \ldots b_{r} \sigma\left(s_{n+1}\right) \varepsilon\left(s_{n+1}\right) s_{n}<_{\mathcal{L}} s_{n}<_{\mathcal{L}} \cdots<_{\mathcal{L}} s_{1}\right)
\end{aligned}
$$

by Remark 2.1, since

$$
c\left(b_{1} \ldots b_{r} \sigma\left(s_{n+1}\right) \varepsilon\left(s_{n+1}\right) s_{n}\right)=c\left(\varepsilon\left(s_{n+1}\right) s_{n}\right)
$$

Hence

$$
\begin{aligned}
\left(b_{1}\right) \ldots\left(b_{r}\right)\left(\sigma\left(s_{n+1}\right)\right)\left(\varepsilon\left(s_{n+1}\right)\right) & \left(s_{n}<_{\mathcal{L}} \cdots<_{\mathcal{L}} s_{1}\right) \\
= & \left(s\left(s_{n+1}\right) \sigma\left(s_{n+1}\right) \varepsilon\left(s_{n+1}\right) s_{n}<_{\mathcal{L}} s_{n}<_{\mathcal{L}} \cdots<_{\mathcal{L}} s_{1}\right) \\
= & \left(s_{n+1}<_{\mathcal{L}} s_{n}<_{\mathcal{L}} \cdots<_{\mathcal{L}} s_{1}\right) \quad \text { by }(4.5 .2)
\end{aligned}
$$

Therefore, by the induction hypothesis, $s \in \hat{S}_{A}^{L}$.

Proof of Lemma 4.6. (i) Let $V=V_{2}$. If $|c(u)|=1$, then the statement is trivially true, since $\left|c_{1}(V)\right|=1$, for all $V \in \mathcal{L B}$. Suppose the statement holds for $n \geq 1$ and let $u \in A^{+}$be such that $|c(u)|=n+1$.

Fix $z \in A^{+}$such that $|c(z)|=n$. Then we have

$$
\begin{aligned}
\left.\left|\left(u \leq_{\mathcal{L}} s_{n} \leq_{\mathcal{L}} \cdots \leq_{\mathcal{L}} s_{1}\right): s_{i} \in A^{+},\right| c\left(s_{i}\right) \mid=i, i \in[1, n]\right\} \mid \\
\quad=\left|\left\{v \in A^{+}: u \leq_{\mathcal{L}} v,|c(v)|=n\right\}\right| . \mid\left\{\left(z \leq_{\mathcal{L}} s_{n-1} \leq_{\mathcal{C}} \cdots \leq_{\mathcal{C}} s_{1}\right): s_{i} \in A^{+},\left|c\left(s_{i}\right)\right|=i,\right. \\
\quad i \in[1, n-1]\} \mid
\end{aligned}
$$

Now

$$
\left|\left\{v: u \leq_{c} v,|c(v)|=n\right\}\right|=(n+1) c_{n}\left(V_{2}\right)
$$


and the induction hypothesis yields that

$$
\left.\|\left(z \leq_{\mathcal{L}} s_{n-1} \leq_{\mathcal{L}} \cdots \leq_{\mathcal{L}} s_{1}\right): s_{i} \in A^{+},\left|c\left(s_{i}\right)\right|=i, i \in[1, n-1]\right\} \mid=\frac{c_{n}\left(\bar{V}_{3}\right)}{c_{n}\left(V_{2}\right)} .
$$

Hence

$$
\left.\left|\left(u \leq_{\mathcal{C}} s_{n} \leq_{\mathcal{C}} \cdots \leq_{\mathcal{L}} s_{1}\right): s_{i} \in A^{+},\right| c\left(s_{i}\right) \mid=i, i \in[1, n]\right\} \mid=\frac{c_{n+1}\left(\bar{V}_{3}\right)}{c_{n+1}\left(V_{2}\right)} .
$$

(ii) Let $V=V_{k}, k$ odd, $k \geq 3$.

If $|c(u)|=1$, the statement is trivially true. Suppose the statement holds for $n \geq 1$ and let $u \in A^{+}$be such that $|c(u)|=n+1$. Fix $z \in A^{+}$such that $|c(z)|=n$. Then we have

$$
\begin{aligned}
& \left|\left(u \leq_{\mathcal{L}} s_{n} \leq_{\mathcal{L}} \cdots \leq_{\mathcal{L}} s_{1}\right): s_{i} \in A^{+},\right| c\left(s_{i}\right) \mid=i, \bar{i}_{k-1}(e(u))=\bar{i}_{k-1}(v), \\
& \left.\quad i_{k-1}\left(e\left(s_{i}\right)\right)=\bar{i}_{k-1}\left(s_{i-1}\right), i \in[1, n]\right\} \mid \\
& =\left|\left\{v: u \leq_{\mathcal{L}} v,|c(v)|=n, \bar{i}_{k-1}(e(u))=\bar{i}_{k-1}(v)\right\}\right| . \\
& \mid\left\{\left(z \leq_{\mathcal{L}} s_{n-1} \leq_{\mathcal{L}} \cdots \leq_{\mathcal{L}} s_{1}\right): s_{i} \in A^{+},\left|c\left(s_{i}\right)\right|=i, \bar{i}_{k-1}\left(e\left(s_{i}\right)\right)=\bar{i}_{k-1}\left(s_{k-1}\right),\right. \\
& \left.\quad i \in[1, n-1], \bar{i}_{k-1}(e(z))=\bar{i}_{k-1}\left(s_{n-1}\right)\right\} \mid
\end{aligned}
$$

Again, the result follows from the next two computations. (See Theorem 2.2.)

$$
\begin{aligned}
& \left|\left\{v: u \leq_{\mathcal{L}} v,|c(v)|=n, \bar{i}_{k-1}(e(u))=\bar{i}_{k-1}(v)\right\}\right| \\
& \quad=\left|\left\{v: \bar{i}_{k-1}(e(u)) \mathcal{L} \bar{i}_{k-1}(v), \bar{i}_{k-1}(e(u))=\bar{i}_{k-1}(v)\right\}\right| \quad \text { by Lemma } 4.6 \\
& \quad=\left|\left\{v: \bar{i}_{k-1}(e(u))=\bar{i}_{k-1}(v)\right\}\right| \\
& \quad=\frac{c_{n}\left(V_{k}\right)}{c_{n}\left(\bar{V}_{k-1}\right)}
\end{aligned}
$$

since there are $c_{n}\left(V_{k}\right)$ words $i_{k}(v)$ with $|c(v)|=n$ and there are $c_{n}\left(\bar{V}_{k-1}\right)$ words $\bar{i}_{k-1}(w)$ with $|c(w)|=n$.

$$
\begin{aligned}
\mid\left\{\left(z \leq_{\mathcal{C}} s_{n-1} \leq_{\mathcal{L}} \cdots \leq_{\mathcal{L}} s_{1}\right): s_{i} \in A^{+},\left|c\left(s_{i}\right)\right|=i, \bar{i}_{k-1}\left(e\left(s_{i}\right)\right)=\bar{i}_{k-1}\left(s_{i-1}\right),\right. \\
\left.\quad i \in[1, n-1], \bar{i}_{k-1}(e(z))=\bar{i}_{k-1}\left(s_{n-1}\right)\right\} \mid \\
=\frac{c_{n}\left(\bar{V}_{k+1}\right)}{c_{n}\left(V_{k}\right)}
\end{aligned}
$$

by the induction hypothesis.

Proof of Lemma 4.7. The proof results immediately from Lemma 4.6. Indeed, if $s=\left(s_{n}<_{\mathcal{L}} s_{n-1}<_{C} \cdots<_{\mathcal{L}} s_{1}\right) \in \hat{S}_{A}^{\mathcal{C}}$, then $\eta_{s, A}(s)=s_{n}$ and the number of elements $s_{n}$ such that $c\left(s_{n}\right)=A_{n}$ is $c_{n}\left(V_{k}\right)$ if $V=V_{k}$. 
Acknowledgement. The author wishes to thank her supervisor Professor John $\mathbf{M}$. Howie for his help and support during the preparation of her thesis.

\section{REFERENCES}

1. J.-C. Birget and J. Rhodes, Iterations of expansions - unambiguous semigroups, J. Pure Appl. Algebra 34 (1984), 1-55.

2. S. Eilenberg, Automata, languages and machines, Vol. B Academic Press, New York, 1976.

3. C. Fennemore, All varieties of bands, Semigroup Forum 44 (1970), 172-179.

4. J. A. Gerhard and M. Petrich, Varieties of bands revisited, Proc. London Math. Soc. (3) 58 (1989), 323-350.

5. L. Polak, On varieties of completely regular semigroups I, Semigroup Forum 32 (1985), 97-123.

6. L. PolaK, On varieties of completely regular semigroups II, Semigroup Forum 36 (1987), 253-284.

7. L. Polak, On varieties of completely regular semigroups III, Semigroup Forum 37 (1988), $1-30$.

8. R. REILLY, The Rhodes expansion and free objects in varieties of completely regular semigroups, J. Pure Appl. Algebra 69 (1990), 89-109.

9. H. Sezinando, Formal languages and idempotent semigroups, Ph.D. thesis, University of St. Andrews, Scotland, 1990.

10. H. Sezinando, The cardinalities of the Green classes of the free objects in varieties of bands, Semigroup Forum 44 (1992), 164-198.

11. B. TILson, Chapters IX and XII in [2].

Centro de Álgebra, Universidade de Lisboa

Av. Prof. Gama Pinto, 2, 1699 lisboa Codex

Portugal

E-mail address: MHELENA@PTMAT.LMC.FC.UL.PT 